\section{RS Ophiuchi - first radio detection of a recurrent nova outburst}

\author{
S. Padin*, R. J. Davis* \& M. F. Bode $†$ \\ * University of Manchester, Nuffield Radio Astronomy Laboratories, \\ Jodrell Bank, Macclesfield, Cheshire SK11 9DL, UK \\ $\dagger$ Department of Astronomy, University of Manchester, \\ Manchester M13 9PL, UK
}

Recurrent novae and classical novae belong to the cataclysmic variable class of objects. Of these, only classical novae outbursts have been hitherto observed at radio wavelengths, and then no earlier than 50 days after the outburst. Here we report the first radio detection of an outburst from a recurrent nova (RS Ophiuchi). The observations begin only 18 days after the optical maximum and show a radio 'light' curve which is different from that of classical novae. The brightness temperature of $\sim 10^{7} \mathrm{~K}$, calculated for this event, greatly exceeds the $10^{4} \mathrm{~K}$ seen in classical novae, and suggests a non-thermal origin for the radio emission from RS Ophiuchi. Although the later emission has a classical $\left(t-t_{0}\right)$ 'light' curve, the radio emission mechanism appears not to turn on until 14 days after the optical nova and takes a further 10 days to reach the $\left(t-t_{0}\right)$ curve. Analogies may be drawn between the radio development of RS Ophiuchi and that of young supernovae.

In both recurrent and classical novae, the outbursts may result from thermonuclear runaway on the surface of a white dwarf in a close binary system, which leads to a rapid increase in luminosity and mass ejection ${ }^{1}$. Based on this model there are two major differences between recurrent and classical novae. First, the secondary component of the binary system in recurrent novae is thought to be a late-type giant rather than a mainsequence dwarf as in classical novae. Second, the inter-outburst timescale is tens of years rather than $10^{4}-10^{5}$ years as predicted for classical novae; for example, the recurrent nova RS Ophiuchi has previously undergone very similar outbursts in 1898,1933 , 1958 and 1967 (ref. 2).

Following the nova outburst reported by Morrison ${ }^{3}$ on 26 January 1985, observations were made with the new Jodrell Bank Mk IA, Mk II 400-MHz bandwidth interferometer system operating at $5 \mathrm{GHz}$ (S.P. and R.J.D., in preparation). This instrument has a baseline of $425 \mathrm{~m}$, giving a lobe spacing of $\sim 30$ arc s at $5 \mathrm{GHz}$. At this frequency, the 4 -arc min beam of the Mk IA telescope results in a typical confusion level of $1 \mathrm{mJy}$. The first flux density measurement of RS Ophiuchi was obtained on 13 February, only 18 days after the outburst. This first detection yielded a flux density of $23 \pm 3 \mathrm{mJy}$ and subsequent observations on 15 February revealed an increase in flux density ${ }^{4}$.

Phase measurements made with the interferometer allow the position of the radio emission to be established to within \pm 2 arc $s$ in right ascension and \pm 4 arc $s$ in declination; within these limits the radio position was found to be consistent with the reported optical position of the nova. Figure 1 shows observations of the radio 'light' curve at $5 \mathrm{GHz}$ for RS Ophiuchi between 13 February and 5 March. Further observations were not possible because of telescope scheduling considerations. The flux density calibration of these data is based ultimately on observations of $3 \mathrm{C} 286$, which is assumed to have a flux density of $7.30 \mathrm{Jy}$ at $5.0 \mathrm{GHz}$ (ref. 5), with the gain-elevation dependence of the interferometer being obtained from observations of many different radio sources. For all these observations, a referencing technique was used with the instrument observing a nearby calibrator source for a short time before moving to the field of interest. Using such a procedure, the flux density of the nova is accurately related to that of the source 1741-038 and this is reflected in the relative error bars in Fig. 1. However, the flux density of 1741-038 is not accurately known and so this has been related to $3 \mathrm{C} 286$. This latter calibration introduces a further systematic error of $\sim 5 \%$ into the flux density scale.

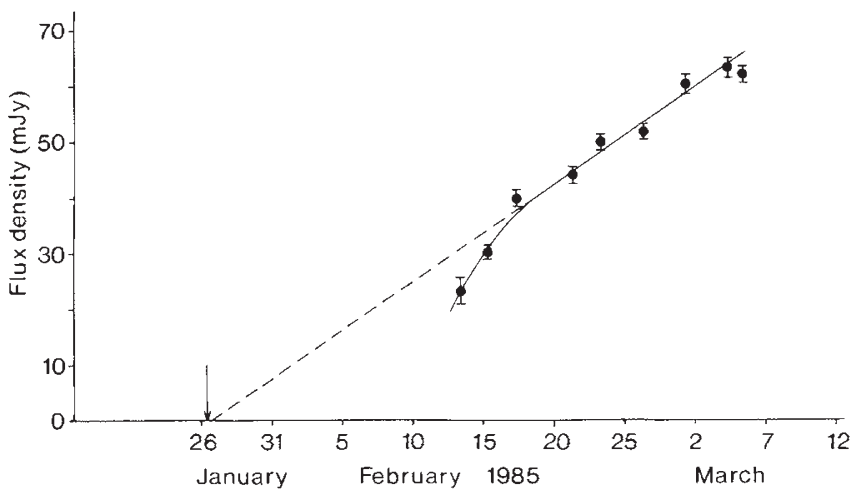

Fig. 1 Radio 'light' curve for the recurrent nova RS Ophiuchi measured at $5 \mathrm{GHz}$ with left-hand circular polarization. The vertical arrow indicates the time of the optical outburst.

Figure 1 clearly shows two phases in the radio development of the nova. Initially there is a rapid increase in flux density at a rate of approximately $4 \mathrm{mJy}$ per day until 18 February; this then gives way to a slower linear increase which projects back to the time of the initial outburst $\left(t_{0}\right)$, giving a $\left(t-t_{0}\right)$ dependence with a constant of proportionality of $1.7 \mathrm{mJy}$ per day. This $\left(t-t_{0}\right)$ dependence is characteristic of the classical radio novae observed by Hjellming et al. $^{6}$. The initial rapid rise indicates that the radio emission 'turned on' about 14 days after the nova explosion, but after a further 10 days it reached the $\left(t-t_{0}\right)$ curve. Thus, although the radio emission was 14 days late in turning on, after $\sim 24$ days the curve proceeds as if the emission had started at $t_{0}$.

Analogies may be drawn between the radio development of RS Ophiuchi and that of Type II supernovae. In the latter case, the non-thermal radio emission is thought to arise from the interaction of an ejected shell with the pre-outburst wind of the late-type supergiant progenitor ${ }^{7}$. Similarly, RS Ophiuchi is thought to eject $\sim 10^{-5} M_{\odot}$ of material at $\sim 1,000 \mathrm{~km} \mathrm{~s}^{-1}$ into pre-existing circumstellar material ${ }^{8}$ which may be associated with a wind from the giant secondary of a binary system.

If we assume a distance of $2 \mathrm{kpc}$ (ref. 9) and an average expansion velocity of $10^{3} \mathrm{~km} \mathrm{~s}^{-1}$, we calculate a brightness temperature of $10^{7} \mathrm{~K}$ for the period of our observations. Initial measurements with the Very Large Array indicate a nearly flat spectrum (R. M. Hjellming, personal communication). Classical novae ${ }^{6}$ exhibit thermal radio emission, typically with a temperature of $10^{4} \mathrm{~K}$, and during the optically thick, rising phase of the light curve have a spectral index of $\sim+1.5$. These differences lead us to suggest a non-thermal origin for the radio emission in RS Ophiuchi. As the $\left(t-t_{0}\right)$ dependence is similar to that of a classical nova, albeit with a different constant of proportionality, these observations suggest that both the optical depth and dynamics of the shell after 24 days may be similar for the two phenomena, even though the radio emission mechanism may differ. The subsequent radio evolution of this system is clearly of considerable interest.

We thank R. M. Hjellring, A. Evans and F. D. Kahn for providing data in advance of publication and for helpful discussions. S.P. and M.F.B. are grateful to the SERC for a Research Studentship and an Advanced Fellowship.

\footnotetext{
Received 11 March: accepted 15 April 1984.

Starrfield, S., Sparks, W. \& Truran, J. Astrophys. J. (in the press).

Warner, B. in Structure and Evolution of Close Binary Systems (eds Eggleton, P. et al) 85-140 (Reidel, Dordrecht, 1973)

3. Morrison, W. IAU Circ. No. 4030 (1985)

4. Padin, S., Davis, R. J. \& Bode, M. F. IAU Circ. No. 4036 (1985).

5. Baars, J. W. M. \& Hartsuijker, A. P. Astr. Astrophys. 17, 172-181 (1972).

6. Hjelliming, R. M., Wade, C. M., Vandenberg, N. R. \& Newell, R. T. Ast. J. 84, 1619-1631 (1979).

Chevalier R. A Astrophys, J. 259, 302-310 (1982)

8. Pottasch, S. R. Bull. astr. Insts Neth. 19, 227-238 (1967)

9. Payne-Gaposhkin, C. in The Galactic Novae, 42 (North-Holland, Amsterdam, 1957).
} 